\title{
The Effect of Exercise Preconditioning on Stroke Outcome in an Experimental Mice Model
}

\section{Soudabeh Naderi, Rahele Ali Mohammadi, Ali Shamsi Zadeh, Masoud Mobini, Fatemeh Amin, Mohammad}

\section{Allahtavakoli*}

Department of Physiology, Pharmacology-Physiology Research Center, Rafsanjan University of Medical Sciences, Rafsanjan, Iran

\section{A BSTRACT}

Introduction: Stroke is a major cause of mortality and long-term disability among adults. The risk of stroke is rapidly increasing in women after menopause. It has been reported that exercise reduces ischemia and reperfusion injury in rat model of stroke. The aim of this study was to investigate the effect of exercise on stroke outcome in the permanent middle cerebral artery occlusion in ovariectomized mice. Materials and Methods: A group of 32 female mice $(25-35 \mathrm{~g})$ were randomly divided into 4 groups as following ( 8 mice in each group): ovariectomy+stroke, stroke, ovariectomy+exercise+stroke, and sham. Seven days before exercise preconditioning, mice were ovariectomized. The exercise group was forced to run on a treadmill 5 days per week, for $40 \mathrm{~min} /$ day at a speed of $18 \mathrm{~m} / \mathrm{min}$ for four weeks. Stroke was induced by permanent middle cerebral artery occlusion (MCAO) method five weeks after ovariectomy. The infarct volume, sensory-motor deficits, and neurological deficits were studied. Results: Infarct volume in the ovariectomy+exercise+stroke and stroke groups was significantly smaller compared to ovariectomy+stroke group. In ovariectomy+exercise+stroke and stroke groups, neurological deficits were significantly lower than ovariectomy+stroke gorup, respectively. sensory-motor deficits were also lower in the ovariectomy+exercise+stroke and stroke groups compared to ovariectomy+stroke group. Conclusion: The present data suggest that exercise preconditioning plays a neuroprotective role in ovariectomized animals and improves stroke outcome in a permanent model of MCAO.

\section{Key words:}

1. Stroke

2. Exercise

3. Ovariectomy

4. Sensorimotor Cortex

5. Gonadal Steroid Hormones

* Corresponding Author: Mohammad Allah Tavakoli

E-mail: m_alahtavakoli@rums.ac.ir 


\title{
اثر يِش شرطىسازى با ورزش بر يِيامدهاى سكتهُ مغزى در يك مدل موشى آزمايشگاهى
}

\author{
سودابه نادرى، راحله على محمدى، على شمسى زاده، مسعود مبينى، فاطمه امين، محمد الله توكلى" \\ كروه فيزيولوزى، مركز تحقيقات فيزيولوزى و فارماكولوزى، دانشكاه علوم يزشكى رفسنجان، رفسنجان، ايران
}

كليد وازهها:

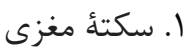

r ب.

r.

أ. قشر حسى -حر كتى

ه. هورمونهاى استروئيدى خناد
مقدمه: سكتهُ مغزى يكى از علل عمده مرگ و مير و ناتوانى هاى طولانى مدت در بزر گسالان است. خطر

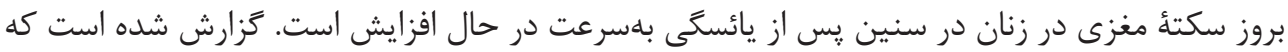

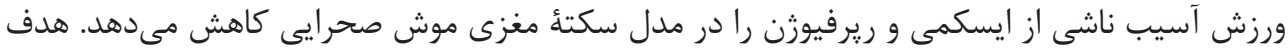

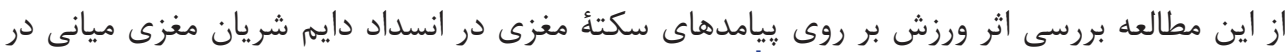

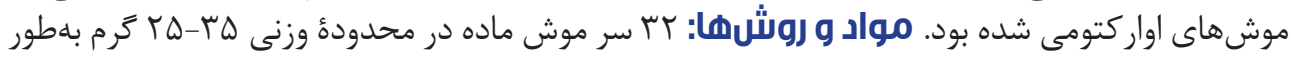

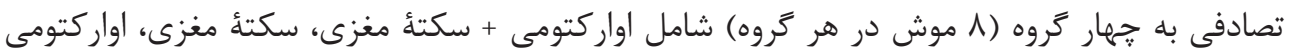

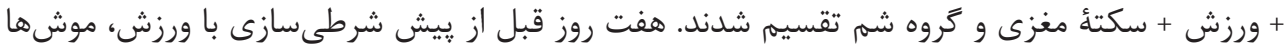

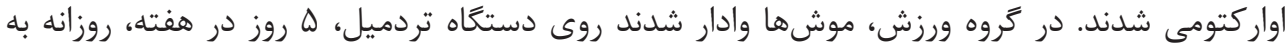

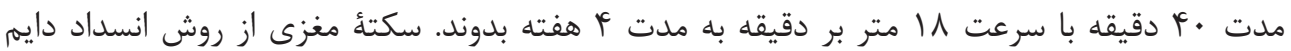

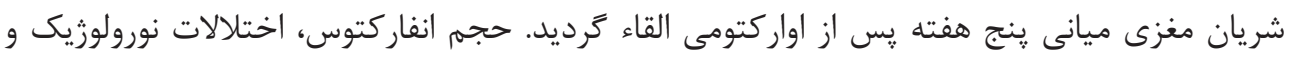

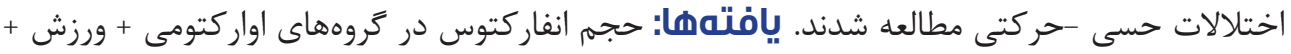

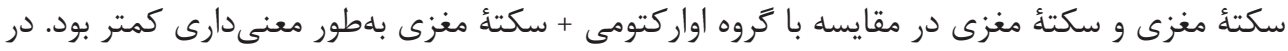

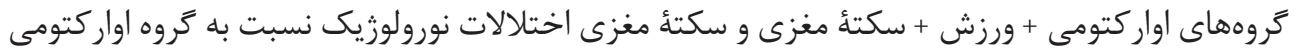

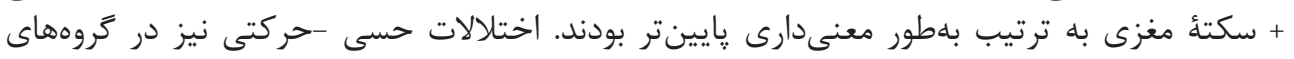

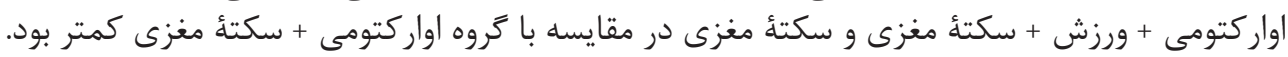

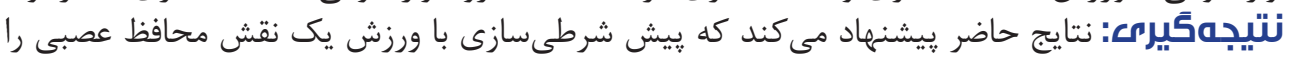

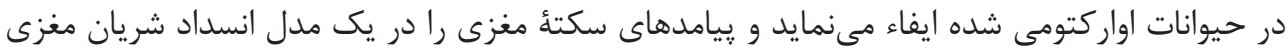
ميانى بهبود مئبخشد.

* نويسنده مسئول: محمد الله توكلى " آدرس الكترونيكى:m_alahtavakoli@rums.ac.ir 
در اين مطالعه از ب T سر موش سورى ماده در محدوده

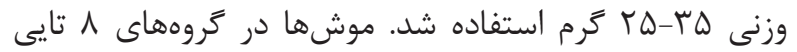

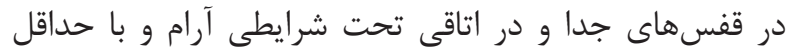

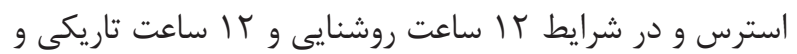

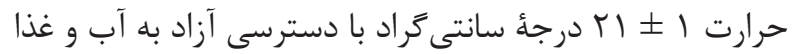

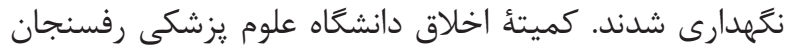
انجام مطالعهُ مذكور را تأييد نمود.

حيوانات بهطور تصادفى به جهار زروه 1 تايى تقسيم شدند:

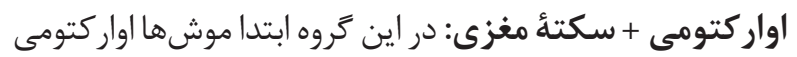

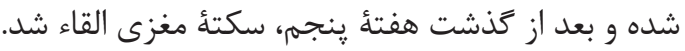

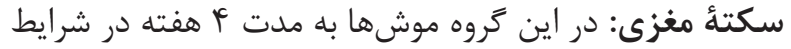

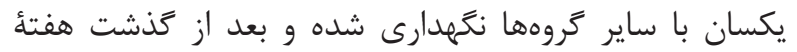
ينجم، سكته مغزى القاء شد.

اواركتومى + ورزش + سكتةُ مغزى: در اين گروه ابتدا

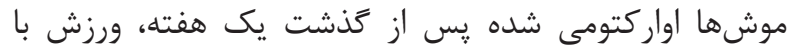

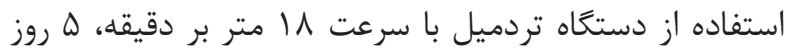

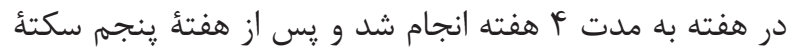
مغزى القاء گرديد. شم: گروهى كه تحت استرس جراحى قرار گرفتند و تمام

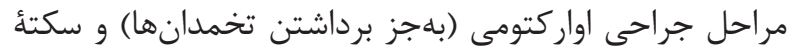

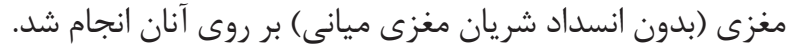

\section{روش ايجاد اواركتومى (القاء يائسگى)}

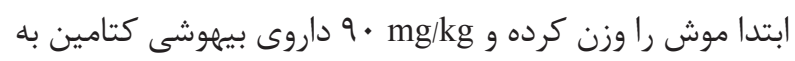

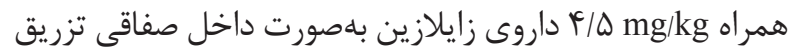
شد و بعد از بيهوشى كامل، ناحية شكمى حئ حيوان ران را تراشيده

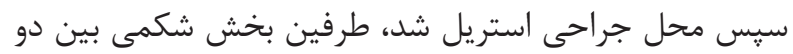

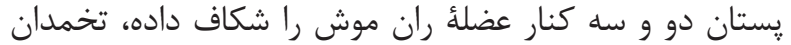

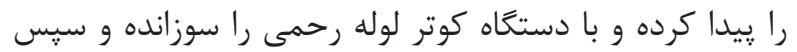

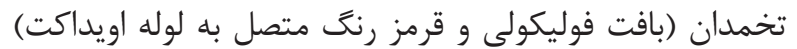

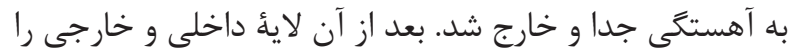

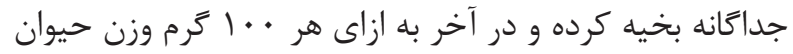

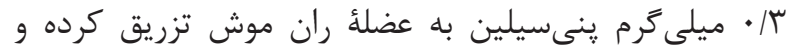
حيوان به قفس برگردانده شد (IV) (IV). ارزيابى صحت انجام اواركتومى

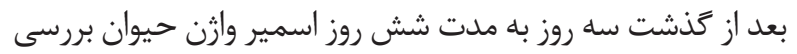

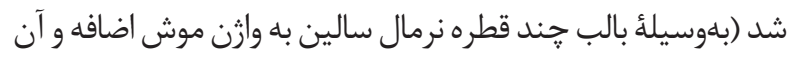

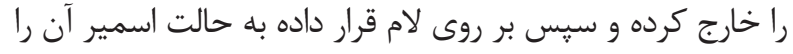

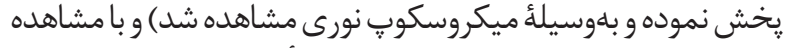
نشدن طرح سرخسى، جراحى اوار كتومى تأييد گرديد (IV).
سكتأه مغزى، سومين عامل مرگ و مير در كشورهاى بيشرفته

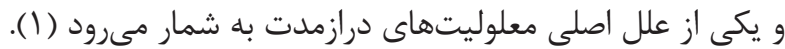

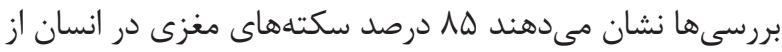

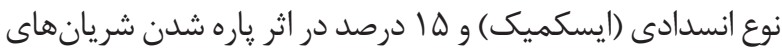

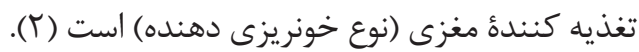

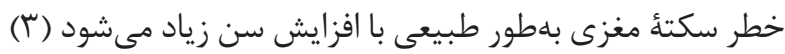

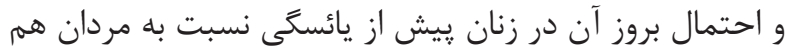

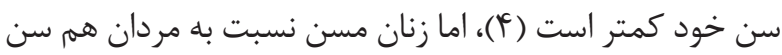

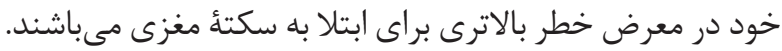

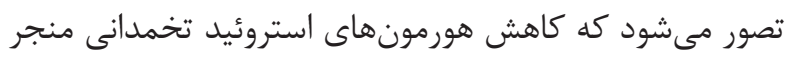

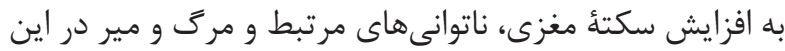

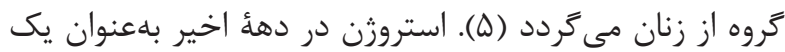

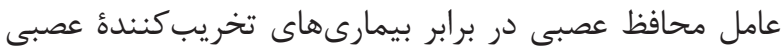

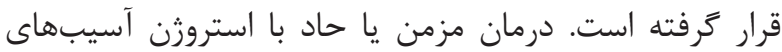
نورونى را در هر دو جنس كاهش مى إنى درند (؟).

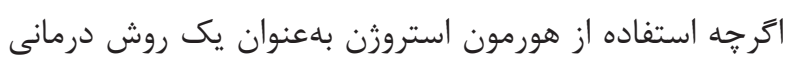

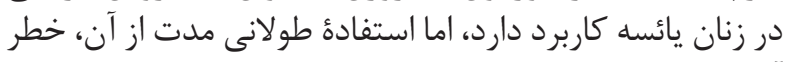

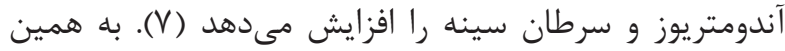

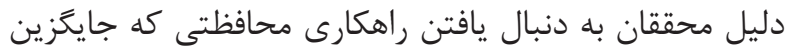

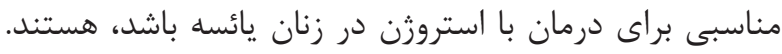

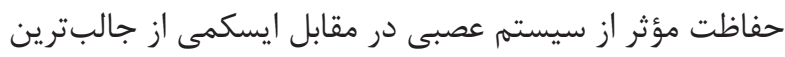

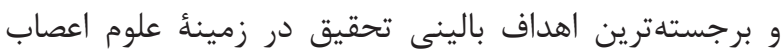

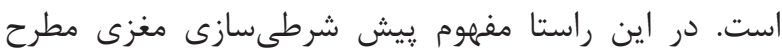

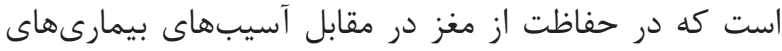

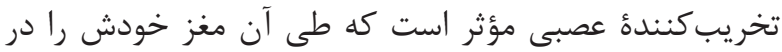

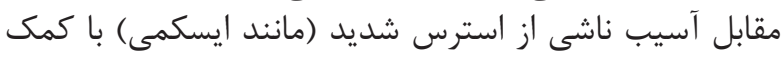

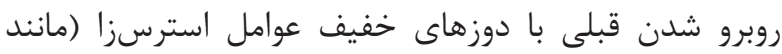

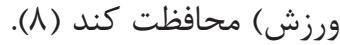

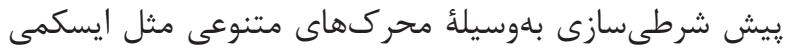

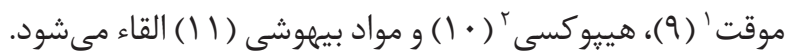

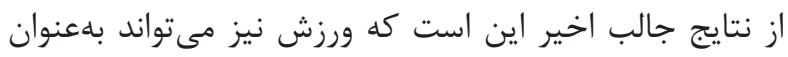

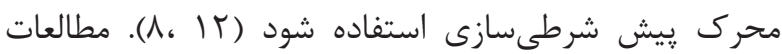

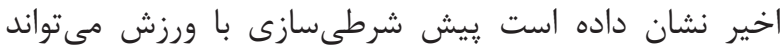

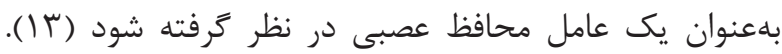

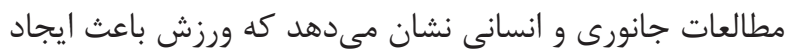

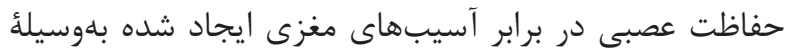

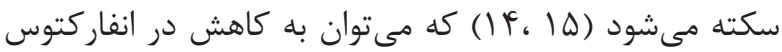

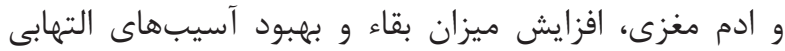

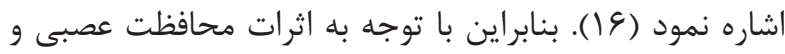

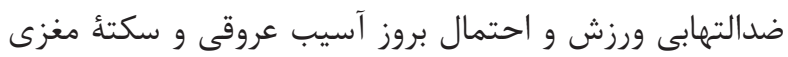

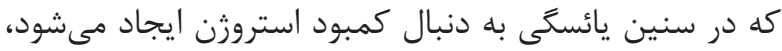

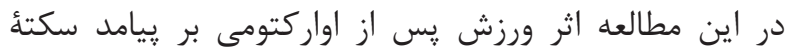
مغزى مورد بررسى قرار زرفت. 
فرمول تعيين حجم انفاركتوس

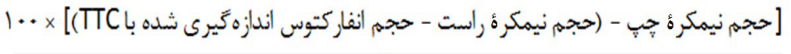

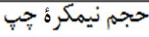

بررسى اختلالات نورولوزيك

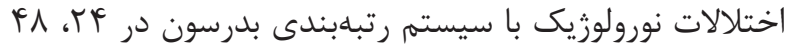

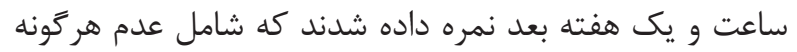

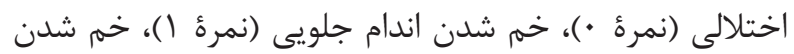

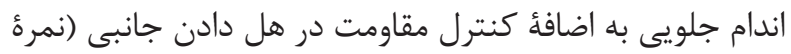
r)

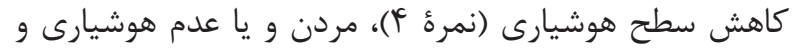

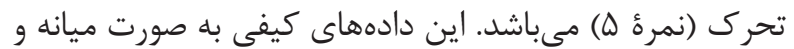

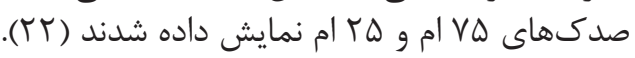

$$
\text { بررسى اختلالات حسى -حركتى }
$$

بلمنظور بررسى عملكرد حسى-حركتى ناشى از القاء سكته

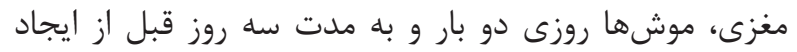

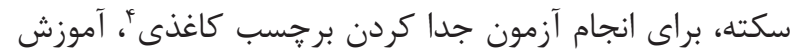

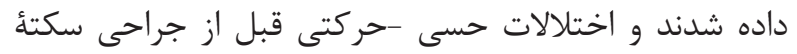

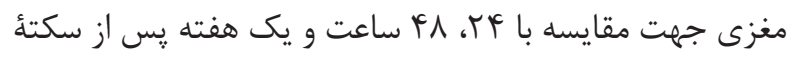

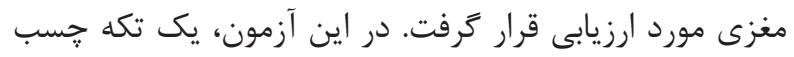

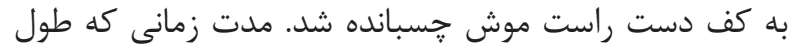

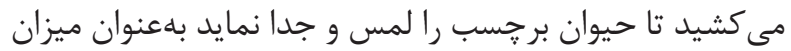

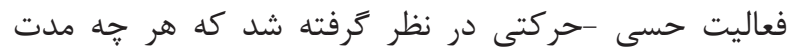

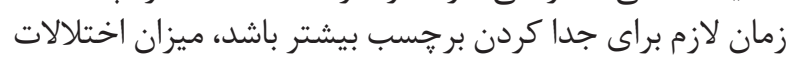

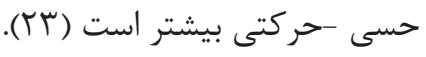

آناليز آمارى تحسى

در مطالعة حاضر از نرمافزار 18 SPSS براى آناليز دادهها

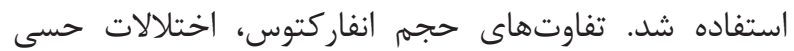

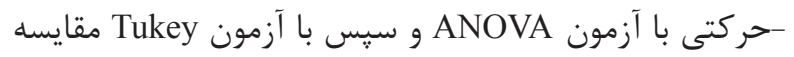

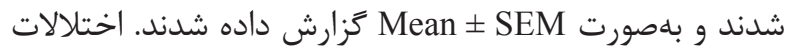

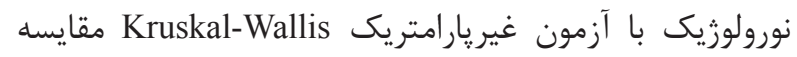

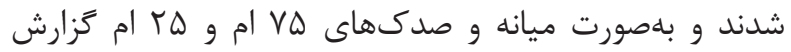

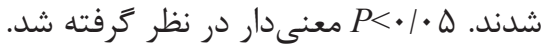

يافتهها

بررسى نتايج حاصل از اندازهَيرى حجم انفار كتوس بافت مغز

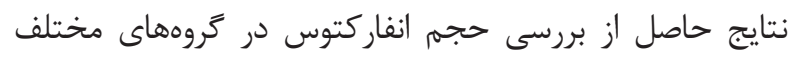

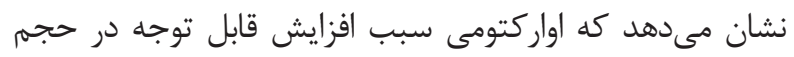

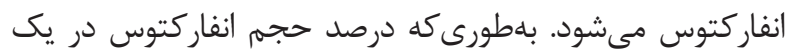

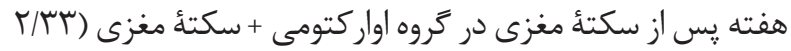

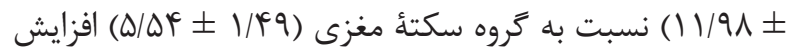

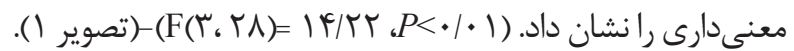

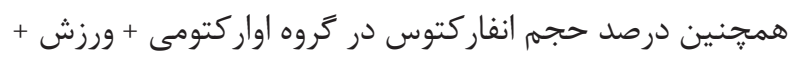

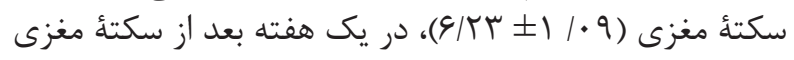

ورزش جسمانى

براى ورزش حيوانات از دستخاه تردميل (دو برقى)-(ساخت كميانى

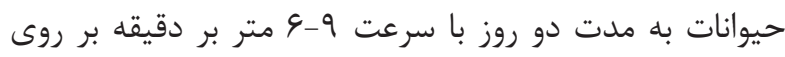

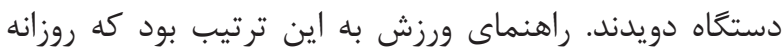

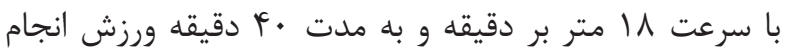

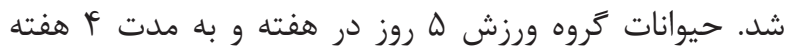

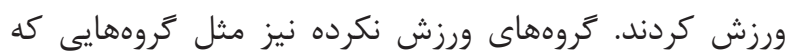

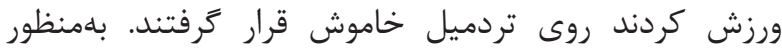

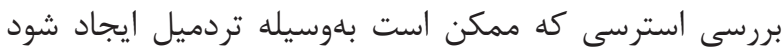

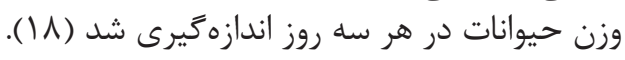

روش ايجاد سكتئ مغزى ميول

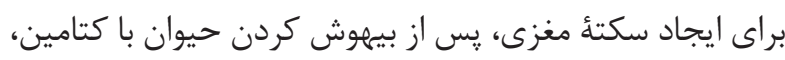

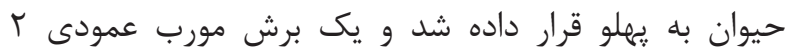

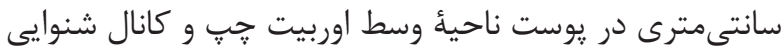

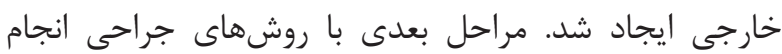

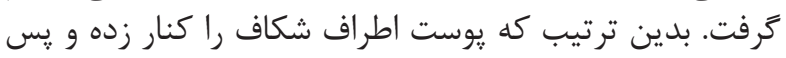

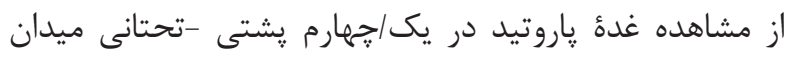

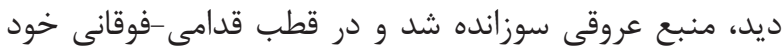

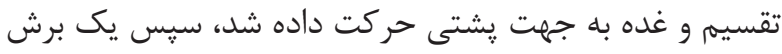

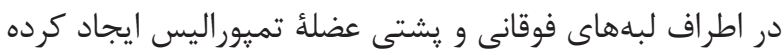

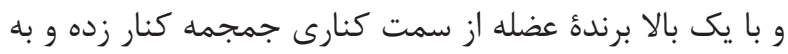

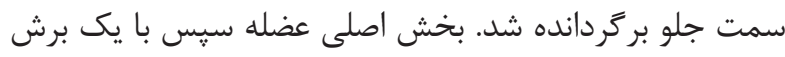

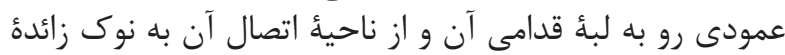

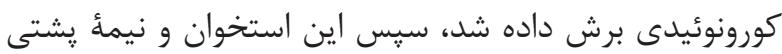

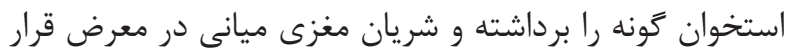

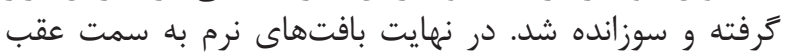

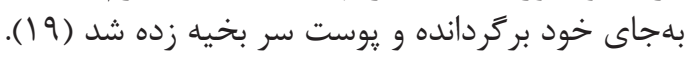

$$
\text { تعيين حجم انفار كتوس حود كردان }
$$

حجم انفار كتوس يكى هفته يس إز سكتأ مغزى اندازهيرى شدر.

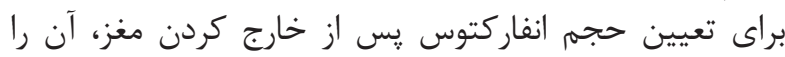

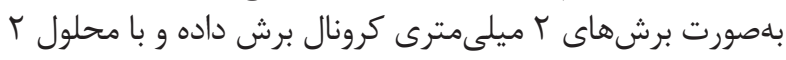

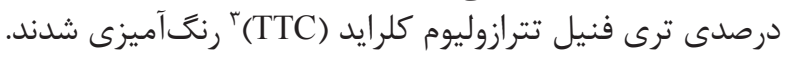

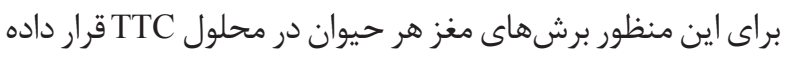

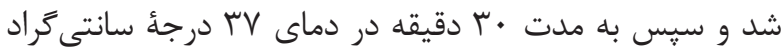

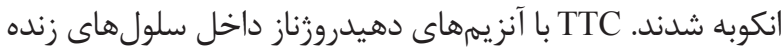

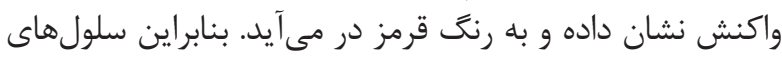

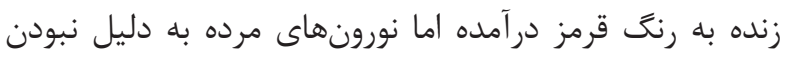

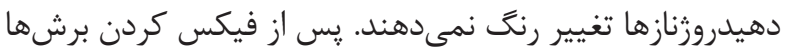

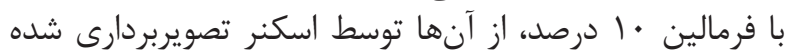

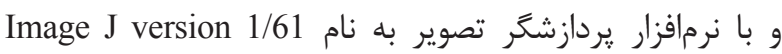

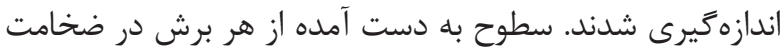

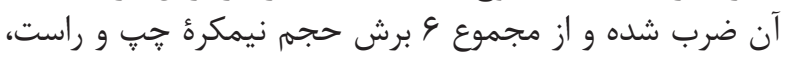

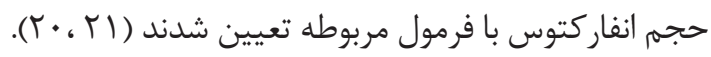


گروه شم كه شامل حيوانات سالم بود، رتبهبندى اختلالات نورولوزيك با سيستم بدرسون در آن صفر بود. بررسى نتايج حاصل از سنجش فعاليت حسى -حر كتى

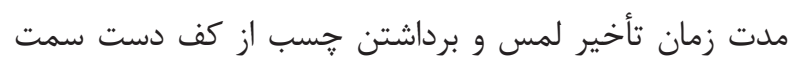

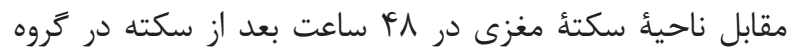

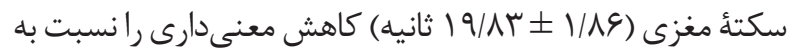

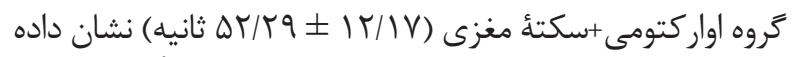

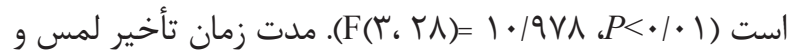

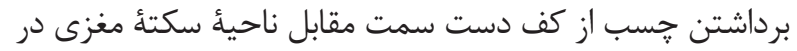
يك هفته بعد از سكته، در كروه سكتئ مغزى (

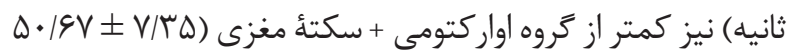

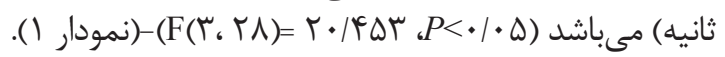

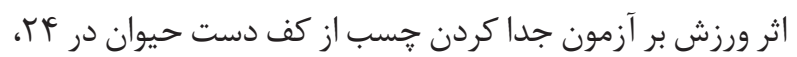

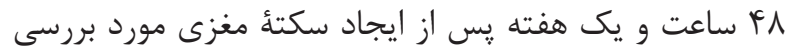

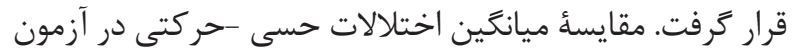

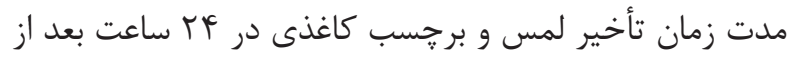

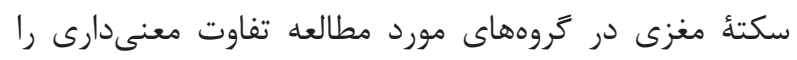

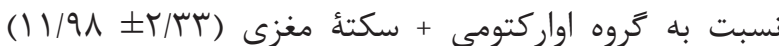

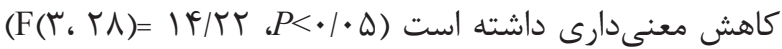

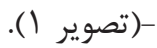

بررسى نتايج حاصل از ارزيابى اختلالات نورولوزيك

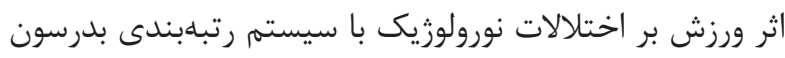

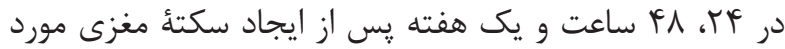

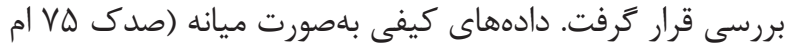

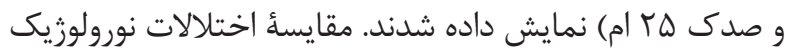

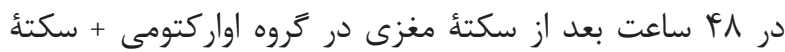

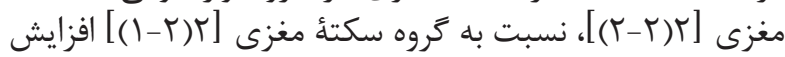

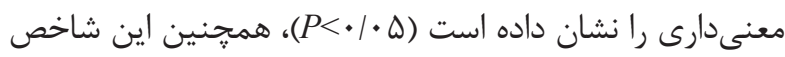

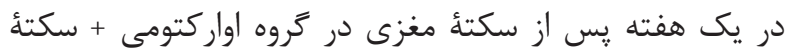

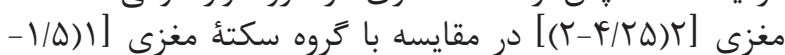

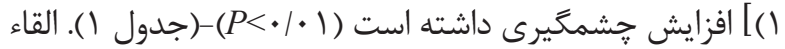

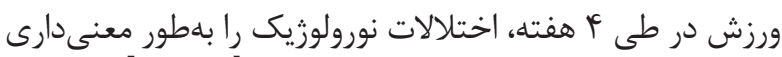
در كروه اواركتومى + ورزش

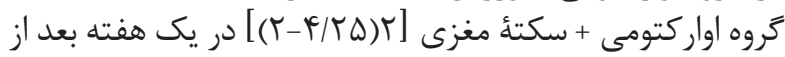

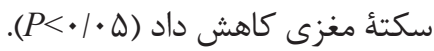

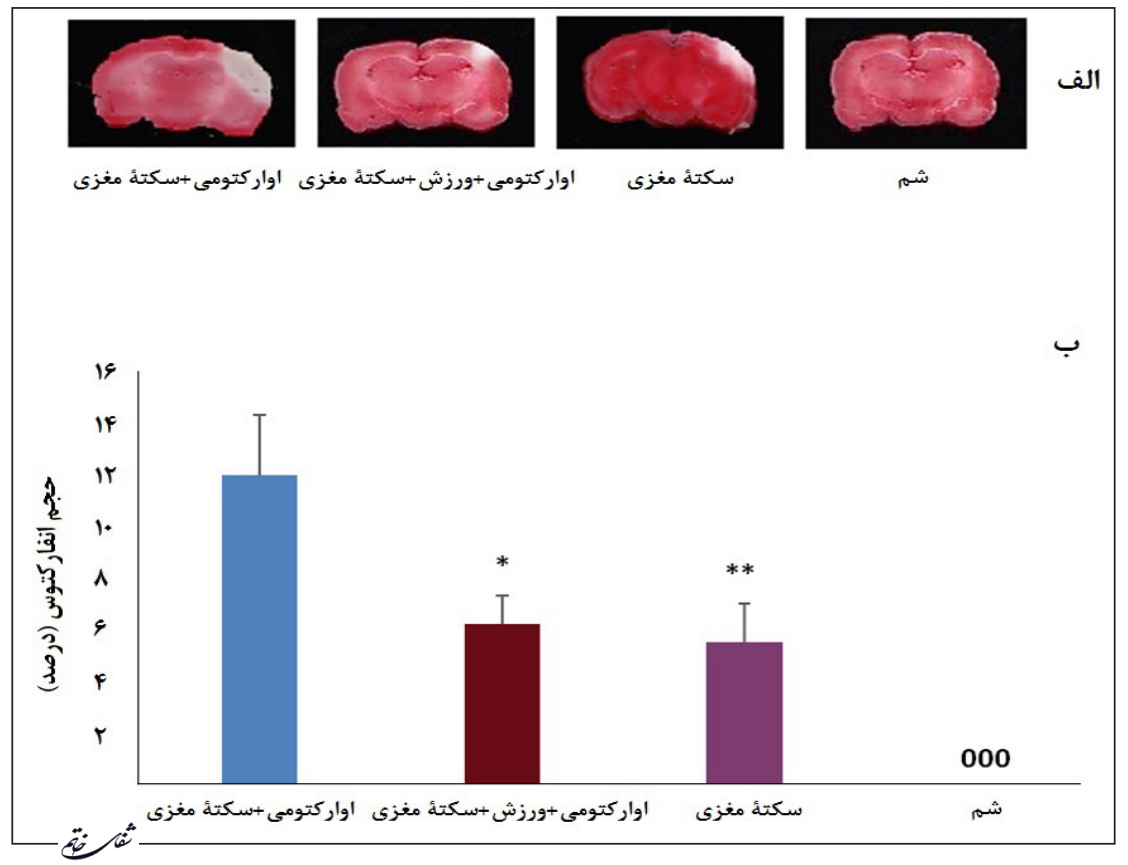

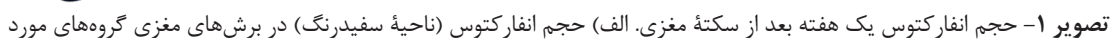

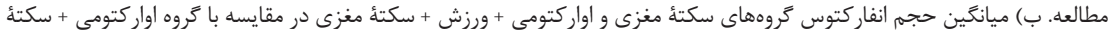

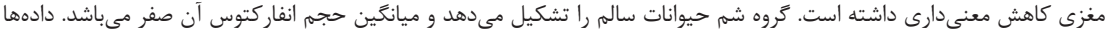

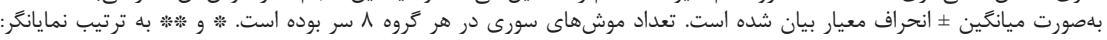

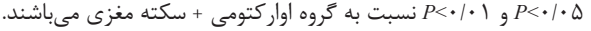

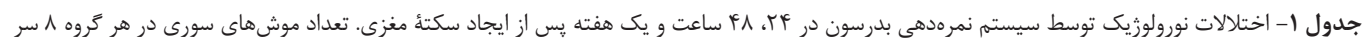

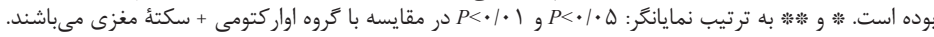

\begin{tabular}{|c|c|c|c|c|}
\hline 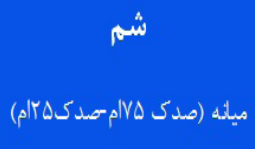 & اواركتوعى +ورزش +سكتة منزى & 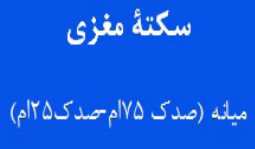 & 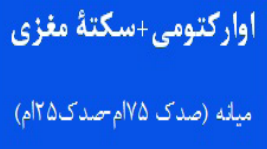 & مان \\
\hline$(\cdot-\cdot) \cdot$ & $(\mid-r) \mid$ & $(\mid-r) \mid$ & $(1 / r \Delta-r) r$ & \\
\hline$(\cdot-\cdot) \cdot$ & $(\mid-r) \mid$ & $*(1-r) Y$ & $(Y-Y) Y$ & FA ساعت \\
\hline$\theta^{+(+-\cdot) \cdot}$ & $*(\mid-Y) \mid$ & 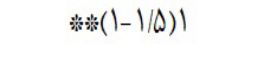 & $(r-r / r \Delta) r$ & | هفته \\
\hline
\end{tabular}




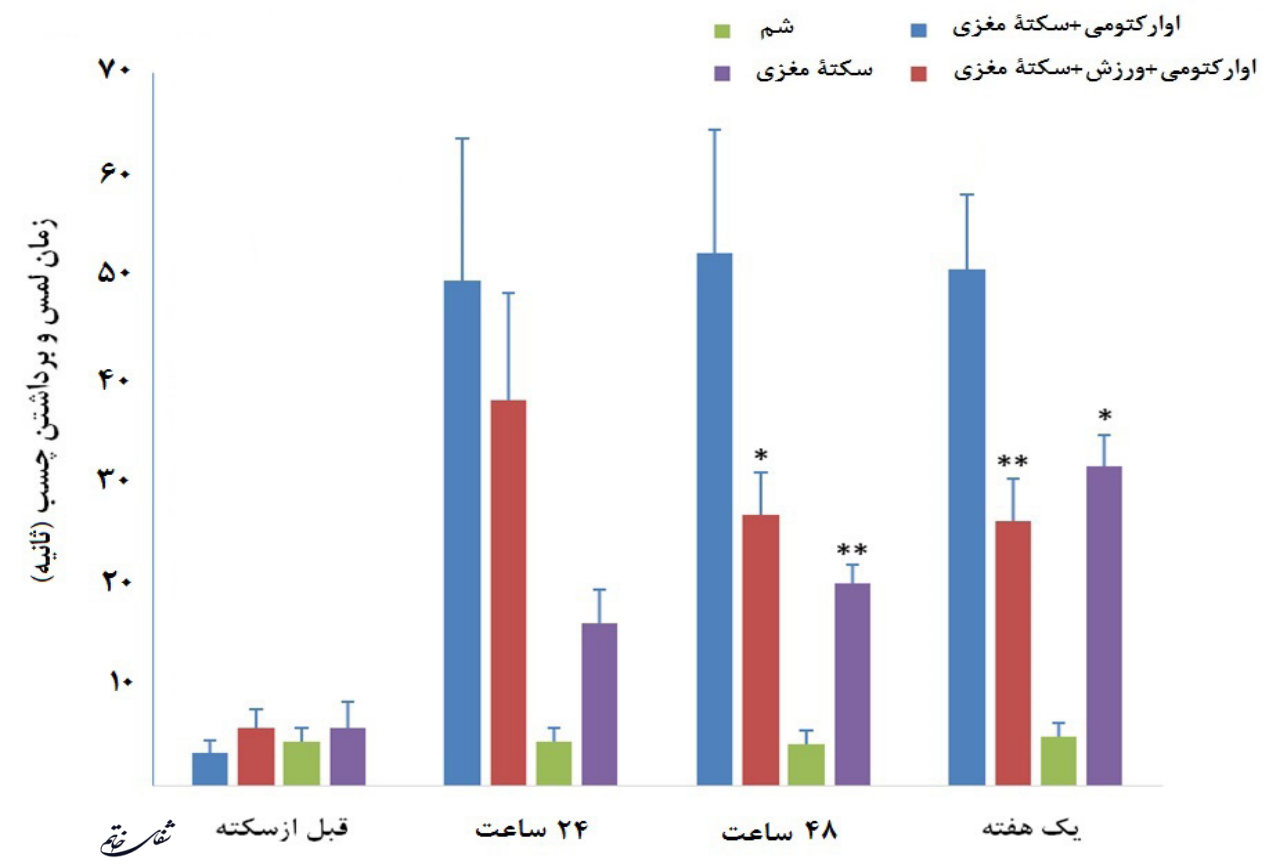

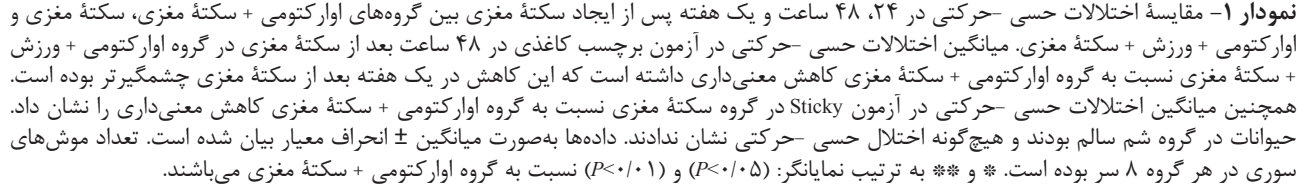

يس از ايجاد سكتأه مغزى، حجم انفاركتوس را بهطور معنى دارى

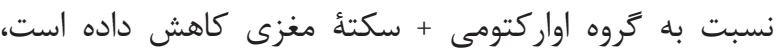

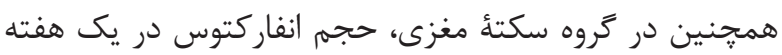

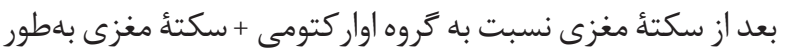

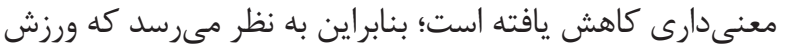

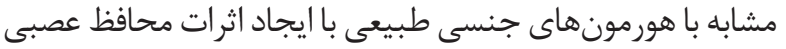
در كاهش ييامدهاى ناشى از سكتهٔ مغزى مؤثر باشد.

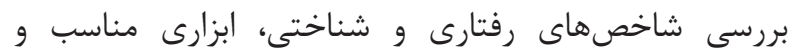

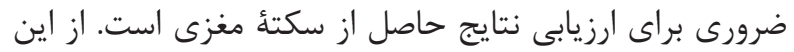

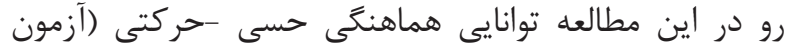

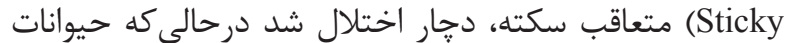

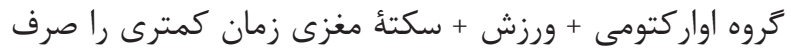

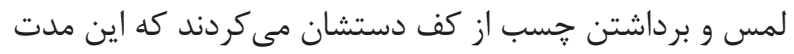

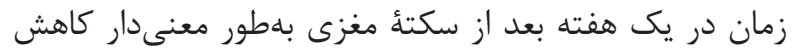

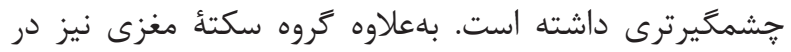

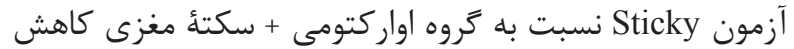

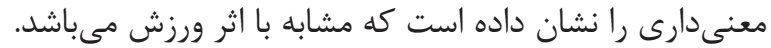

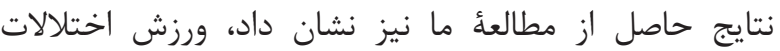

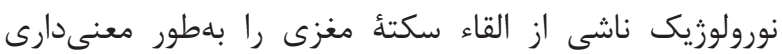

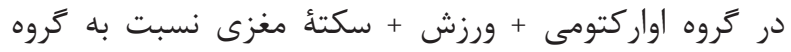
اواركتومى + سكتئ مغزى كاهش دار داده است

بسيارى از مطالعات نشان دادهاند كه استروزن داراى اثر

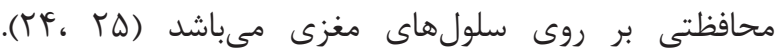

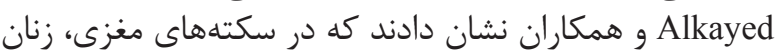

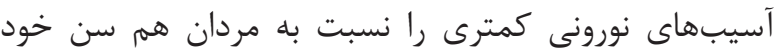

نشان نداده است. مدت زمان تأخير لمس و برداشتن جسب از

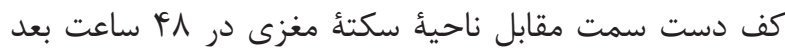

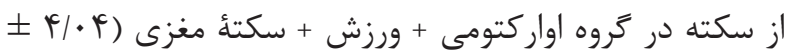
I T//V) ( هن

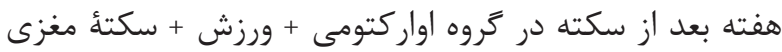

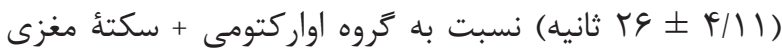

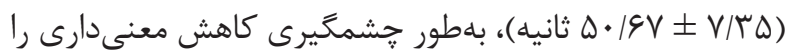

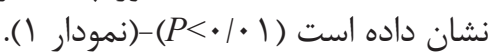
باتوجه به اينكه حيوانات درگروه شم سالم بودند و هيجَّونه

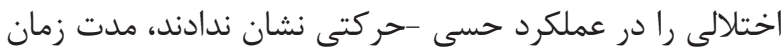

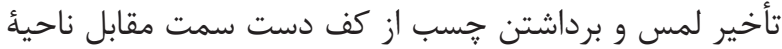

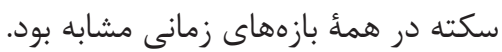

$$
\text { بحث و نتيجه }
$$

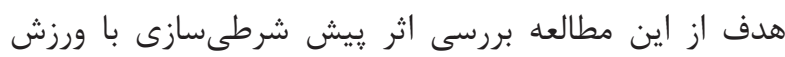

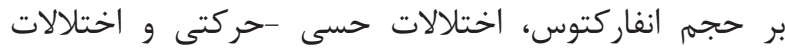

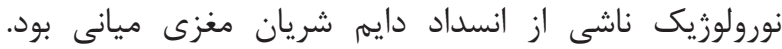

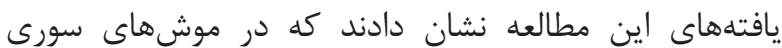

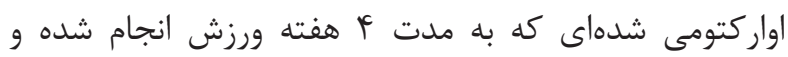

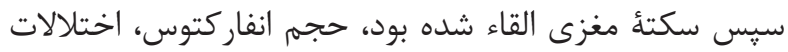

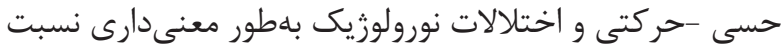

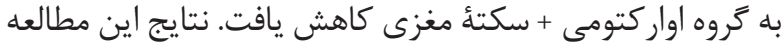

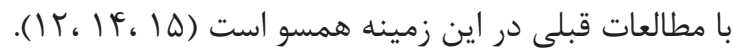
انجام ورزش در موشهاى سورى اواركتومى شده در يك هفته 


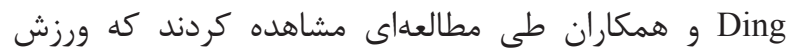

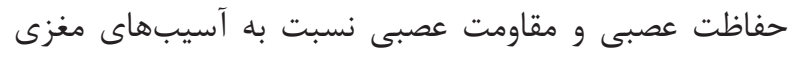

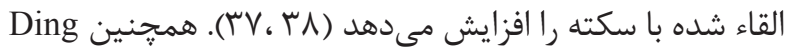

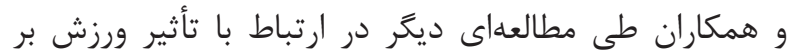

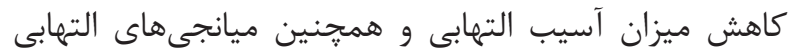

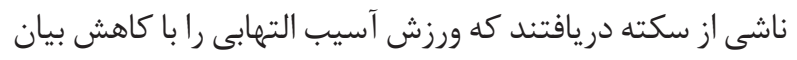

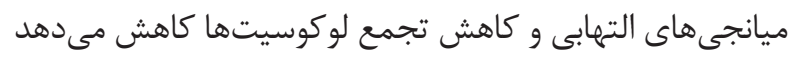

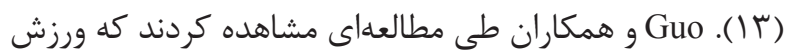

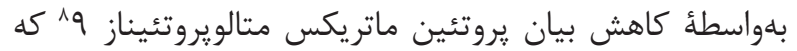

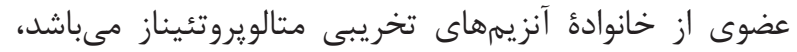

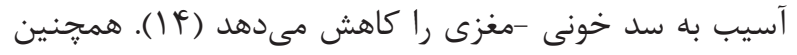

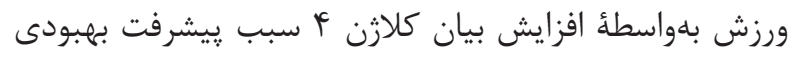

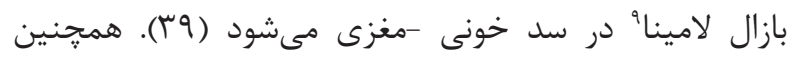

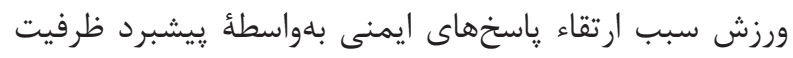

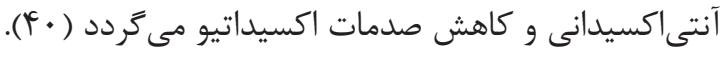
در مجموع نتايج حاصل از اين مطالعه و مطالعات قبلى بر بر إندان

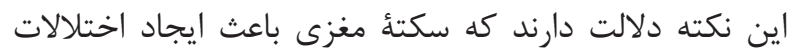

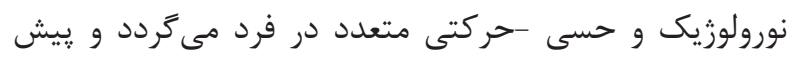

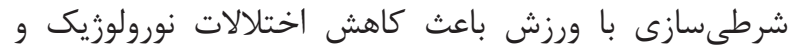

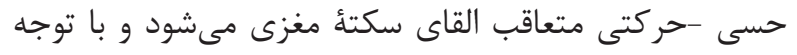

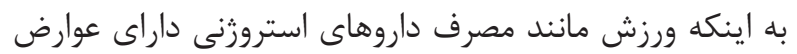

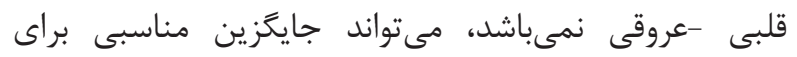

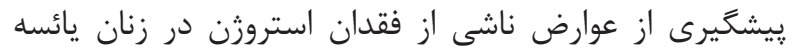

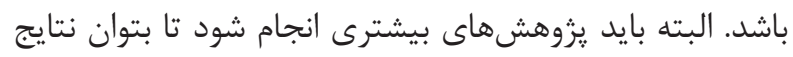

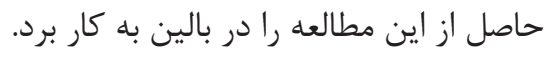

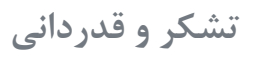

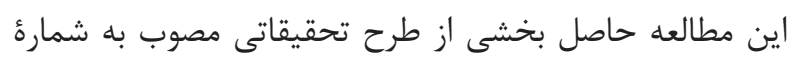

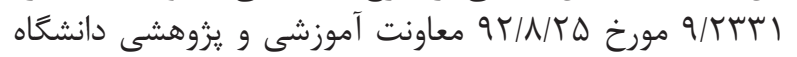

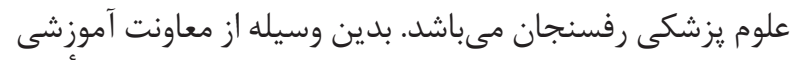

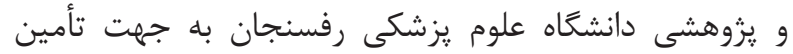

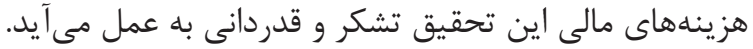

1. Taylor TN, Davis PH, Torner JC, Holmes J, Meyer JW, Jacobson MF. Lifetime cost of stroke in the United States. Stroke. 1996; 27(9): 1459-66.

2. Mergenthaler P, Dirnagl U, Meisel A. Pathophysiology of stroke: lessons from animal models. Metab Brain Dis. 2004; 19(3-4): 151-67.

3. Chinwatanakul S, Boonyapisit K, Pornsriniyom D, Proyoonwiwat N, Senanarong V, Chaisevikul R, et al. Siriraj acute stroke unit: 10 years experience. J Med Assoc Thai. 2012; 95: S235-44.

4. Barrett-Connor E, Bush TL. Estrogen and coronary

${ }^{5}$ Neurotransmitters

${ }^{6}$ Caspase

${ }^{7}$ Low density lipoprotein
متحمل مىشوند (צ) (ب). بررسىهاى ديخرى كه در اين زمينه

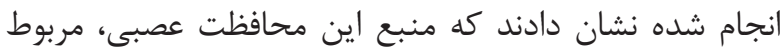

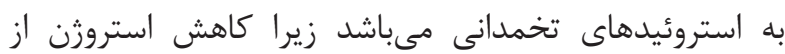

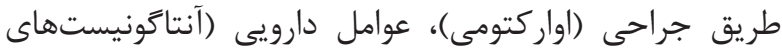

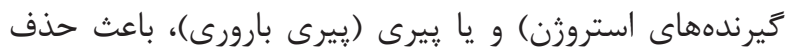

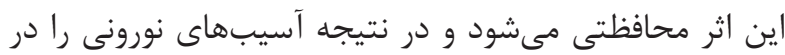

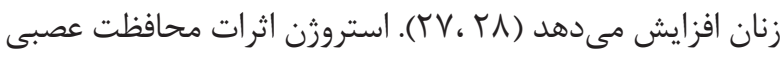

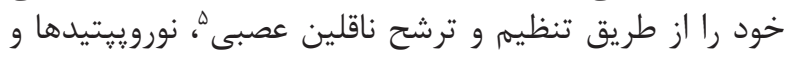

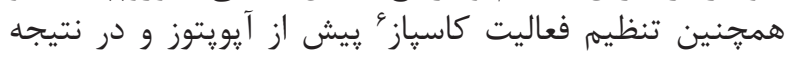

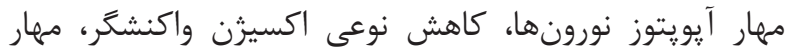

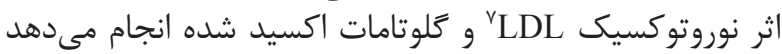

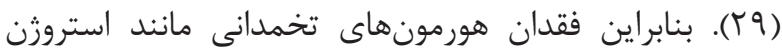

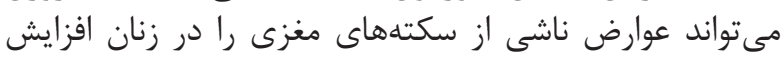

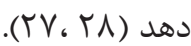

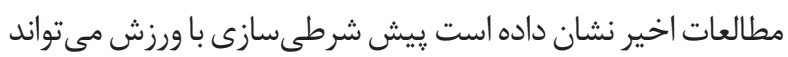

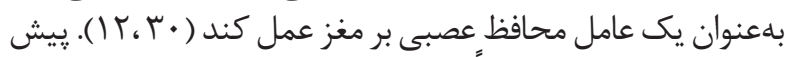

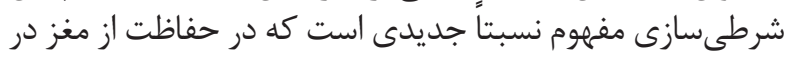

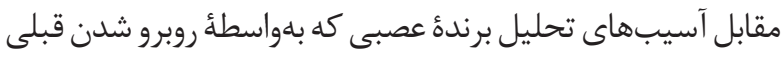

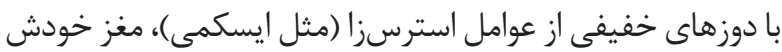

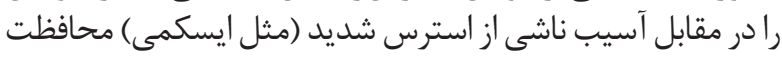

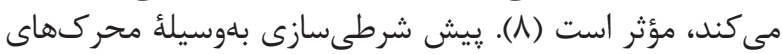

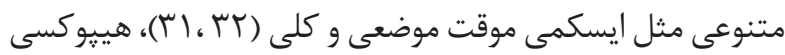

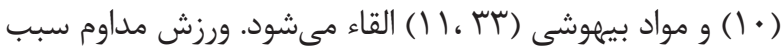

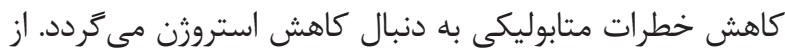

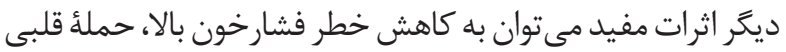

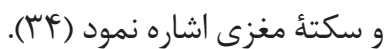

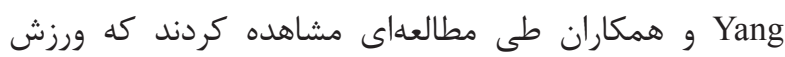

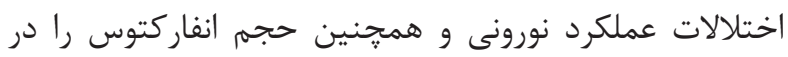

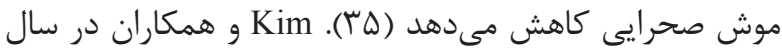

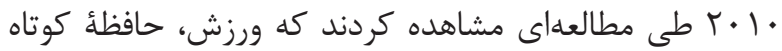

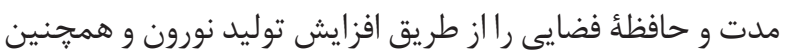

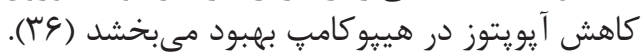

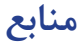

heart disease in women. JAMA. 1991; 265(14): 1861-7.

5. Hochner-Celnikier D, Manor O, Garbi B, ChajekShaul T. Gender gap in cerebrovascular accidents: comparison of the extent, severity, and risk factors in men and women aged 45-65. Int J Fertil Womens Med. 2004; 50(3): 122-8.

6. Wise PM. Estrogens and neuroprotection. Trends Endocrinol Metab. 2002; 13(6): 229-30.

7. Hammond C. Women's concerns with hormone replacement therapy-compliance issues. Fertil Steril. 1994; 62(6 Suppl 2): 157S-60S.

\footnotetext{
${ }^{8}$ Matrix metalloproteinase 9 (MMP-9)

${ }^{9}$ Basal lamina
} 
8. Dirnagl U, Simon RP, Hallenbeck JM. Ischemic tolerance and endogenous neuroprotection. Trends Neurosci. 2003; 26(5): 248-54.

9. Hoshi A, Nakahara T, Kayama H, Yamamoto T. Ischemic tolerance in chemical preconditioning: possible role of astrocytic glutamine synthetase buffering glutamate-mediated neurotoxicity. J Neurosci Res. 2006; 84(1): 130-41.

10. Gidday JM, Shah AR, Maceren RG, Wang Q, Pelligrino DA, Holtzman DM, et al. Nitric oxide mediates cerebral ischemic tolerance in a neonatal rat model of hypoxic preconditioning. J Cereb Blood Flow Metab. 1999; 19(3): 331-40.

11. Kobayashi S, Harris VA, Welsh FA. Spreading depression induces tolerance of cortical neurons to ischemia in rat brain. J Cereb Blood Flow Metab. 1995; 15(5): 721-7.

12. Ding YH, Young CN, Luan X, Li J, Rafols JA, Clark JC, et al. Exercise preconditioning ameliorates inflammatory injury in ischemic rats during reperfusion. Acta Neuropathol. 2005; 109(3): 237-46.

13. Ding YH, Mrizek M, Lai Q, Wu Y, Reyes R, Li J, et al. Exercise preconditioning reduces brain damage and inhibits TNF- $\alpha$ receptor expression after hypoxia/ reoxygenation: an in vivo and in vitro study. Curr Neurovasc Res. 2006; 3(4): 263-71.

14. Guo M, Cox B, Mahale S, Davis W, Carranza A, Hayes $\mathrm{K}$, et al. Pre-ischemic exercise reduces matrix metalloproteinase-9 expression and ameliorates bloodbrain barrier dysfunction in stroke. Neuroscience. 2008; 151(2): 340-51.

15. Feng R, Zhang M, Wang X, Li WB, Ren SQ, Zhang F. Pre-ischemic exercise alleviates oxidative damage following ischemic stroke in rats. Exp Ther Med. 2014; 8(4): 1325-9.

16. Li J, Luan X, Clark JC, Rafols JA, Ding Y. Neuroprotection against transient cerebral ischemia by exercise pre-conditioning in rats. Neurol Res. 2004; 26(4): 404-8.

17. Emerton K, Hu B, Woo A, Sinofsky A, Hernandez C, Majeska RJ, et al. Osteocyte apoptosis and control of bone resorption following ovariectomy in mice. Bone. 2010; 46(3): 577-83.

18. Al-Jarrah MD, Jamous M. Effect of endurance exercise training on the expression of GFAP, S100B, and NSE in the striatum of chronic/progressive mouse model of Parkinson's disease. Neuro Rehabilitation.
2011; 28(4): 359-63.

19. Bogaert-Buchmann A, Poittevin M, Po C, Dupont D, Sebrié C, Tomita Y, et al. Spatial and temporal MRI profile of ischemic tissue after the acute stages of a permanent mouse model of stroke. Open Neuroimag J. 2013; 7: 4-14.

20. Ren C, Gao X, Niu G, Yan Z, Chen X, Zhao H. Delayed postconditioning protects against focal ischemic brain injury in rats. PLoS One. 2008; 3(12): e3851. doi: 10.1371/journal.pone.0003851.

21. Jiao H, Wang Z, Liu Y, Wang P, Xue Y. Specific role of tight junction proteins claudin-5, occludin, and ZO-1 of the blood-brain barrier in a focal cerebral ischemic insult. J Mol Neurosci. 2011; 44(2): 130-9.

22. Allahtavakoli M, Kahnouei $\mathrm{MH}$, Rezazadeh $\mathrm{H}$, Roohbakhsh A, Mahmoodi MH, Moghadam-Ahmadi A, et al. Delayed combination therapy of local brain hypothermia and decompressive craniectomy on acute stroke outcome in rat. Iran J Basic Med Sci. 2014; 17(7): 476-82.

23. Micale V, Marco Leggio G, Mazzola C, Drago F. Cognitive effects of SL65. 0155, a serotonin 5-HT4 receptor partial agonist, in animal models of amnesia. Brain Res. 2006; 1121(1): 207-15.

24. Bushnell CD. Stroke in women: risk and prevention throughout the lifespan. Neurol Clin. 2008; 26(4): 1161-76.

25. Krause DN, Duckles SP, Pelligrino DA. Influence of sex steroid hormones on cerebrovascular function. J Appl Physiol. 2006; 101(4): 1252-61.

26. Alkayed NJ, Harukuni I, Kimes AS, London ED, Traystman RJ, Hurn PD. Gender-linked brain injury in experimental stroke. Stroke. 1998; 29(1): 159-66.

27. Alkayed NJ, Murphy SJ, Traystman RJ, Hurn PD, Miller VM. Neuroprotective effects of female gonadal steroids in reproductively senescent female rats. Stroke. 2000; 31(1): 161-8.

28. Murphy SJ, McCullough LD, Smith JM. Stroke in the female: role of biological sex and estrogen. ILAR J. 2004; 45(2): 147-59.

29. Fiocchetti M, Ascenzi P, Marino M. Neuroprotective effects of $17 \beta$-estradiol rely on estrogen receptor membrane initiated signals. Front Physiol. 2012; 3: 73. doi: 10.3389/fphys.2012.00073.

30. Liebelt B, Papapetrou P, Ali A, Guo M, Ji X, Peng C, et al. Exercise preconditioning reduces neuronal apoptosis 
in stroke by up-regulating heat shock protein-70 (heat shock protein-72) and extracellular-signal-regulatedkinase 1/2. Neuroscience. 2010; 166(4): 1091-100.

31. Dillon GM, Qu X, Marcus JN, Dodart JC. Excitotoxic lesions restricted to the dorsal CA1 field of the hippocampus impair spatial memory and extinction learning in C57BL/6 mice. Neurobiol Learn Mem. 2008; 90(2): 426-33.

32. Kunz A, Park L, Abe T, Gallo EF, Anrather J, Zhou $\mathrm{P}$, et al. Neurovascular protection by ischemic tolerance: role of nitric oxide and reactive oxygen species. J Neurosci. 2007; 27(27): 7083-93.

33. Zhao P, Zuo Z. Isoflurane preconditioning induces neuroprotection that is inducible nitric oxide synthasedependent in neonatal rats. J Anaesthesiol. 2004; 101(3): 695-703.

34. Mishra N, Mishra VN, Devanshi. Exercise beyond menopause: Dos and Don'ts. J Midlife Health. 2011; 2(2): 51-6.

35. Yang R, Cui HJ, Wang H, Wang Y, Liu JH, Li Y, et al. N-Stearoyltyrosine protects against glutamateinduced oxidative toxicity by an apoptosis-inducing factor (AIF)-mediated caspase-independent cell death pathway. J Pharmacol Sci. 2014; 124(2): 169-79.
36. Kim SE, Ko IG, Kim BK, Shin MS, Cho S, Kim CJ, et al. Treadmill exercise prevents aging-induced failure of memory through an increase in neurogenesis and suppression of apoptosis in rat hippocampus. Exp Gerontol. 2010; 45(5): 357-65.

37. Ding YH, Luan XD, Li J, Rafols JA, Guthinkonda M, Diaz FG, et al. Exercise-induced overexpression of angiogenic factors and reduction of ischemia/ reperfusion injury in stroke. Curr Neurovasc Res. 2004; 1(5): 411-20.

38. Ding Y, Li J, Luan X, Ding YH, Lai Q, Rafols $\mathrm{JA}$, et al. Exercise pre-conditioning reduces brain damage in ischemic rats that may be associated with regional angiogenesis and cellular overexpression of neurotrophin. Neuroscience. 2004; 124(3): 583-91.

39. Guo M, Lin V, Davis W, Huang T, Carranza A, Sprague S, et al. Preischemic induction of TNF- $\alpha$ by physical exercise reduces blood-brain barrier dysfunction in stroke. J Cereb Blood Flow Metab. 2008; 28(8): 1422-30.

40. Radák Z, Sasvári M, Nyakas C, Pucsok J, Nakamoto $\mathrm{H}$, Goto $\mathrm{S}$. Exercise preconditioning against hydrogen peroxide-induced oxidative damage in proteins of rat myocardium. Arch Biochem Biophys. 2000; 376(2): 248-51. 